\title{
Ensemble Projection for Semi-supervised Image Classification
}

\author{
Dengxin Dai \\ Computer Vision Lab, ETH Zurich \\ daievision.ee.ethz.ch
}

\author{
Luc Van Gool \\ Computer Vision Lab, ETH Zurich \\ vangoolevision.ee.ethz.ch
}

\begin{abstract}
This paper investigates the problem of semi-supervised classification. Unlike previous methods to regularize classifying boundaries with unlabeled data, our method learns a new image representation from all available data (labeled and unlabeled) and performs plain supervised learning with the new feature. In particular, an ensemble of image prototype sets are sampled automatically from the available data, to represent a rich set of visual categories/attributes. Discriminative functions are then learned on these prototype sets, and image are represented by the concatenation of their projected values onto the prototypes (similarities to them) for further classification. Experiments on four standard datasets show three interesting phenomena: (1) our method consistently outperforms previous methods for semi-supervised image classification; (2) our method lets itself combine well with these methods; and (3) our method works well for self-taught image classification where unlabeled data are not coming from the same distribution as labeled ones, but rather from a random collection of images.
\end{abstract}

\section{Introduction}

Providing efficient solution to image classification has always been a major focus in computer vision. Most of the classification system [3, 17] heavily rely on manually labeled training data, which is expensive and sometimes impossible to acquire. The scarcity of annotations, combined with the explosion of image data, has shifted focus towards learning with less supervision. As a result, numerous techniques such as semi-supervised learning [10], active learning [12], transfer learning [2 4], and self-taught learning [25] have been developed.

In this paper, we are interested in the problem of semisupervised learning (SSL) for image classification. The task is to design a method that can make use of unlabeled images, while learning classifiers from labeled ones. Recent research in SSL has obtained some success in solving this problen $[10,18,21]$. Most of these methods build them- selves upon the local-consistency assumption that data samples with high similarity should share the same label. This assumption allows the geometrical structure of unlabeled data to regularize the classifying functions. While improvements have been reported, these methods share three common drawbacks.

First of all, these methods only exploit the localconsistency assumption in image feature space, and ignore other prior information. Another reasonable assumption borne out by our results - is that samples with very low similarity are in high probability come from different classes. We call this the exotic-inconsistency assumption, and design a method to exploit it also for SSL. Furthermore, most previous methods design specialized learning algorithms to leverage the structure of unlabeled data [2, 1, , 18], so users often need to change their learning methods in order to utilize the cheap unlabeled data. This limits the applicability of SSL, as users usually are reluctant to give up their favorite classifiers. Last but not the least, previous methods assume that the unlabeled data are coming from more or less the same distribution as the labeled data. This imposes restrictions as well, as many applications have no prior access to the data to be classified. To overcome these limitations, we depart from the traditional paradigm and propose another route to SSL in this paper. Below, we present our motivations and outline the method.

People learn and generalize object classes well from their characteristics, such as color, texture, and size. We also do so by comparing an object with other objects in the world. This is part of Eleanor Rosch's prototype theory [27], that states that an object's class is determined by its similarity to prototypes which represent object categories. The theory is suitable for transfer learning [2 4], where labeled data of other categories are available. An important question is whether the theory can also be used for SSL, with its huge amount of unlabeled data. Our paper investigates this problem.

To use this paradigm, we first need to create the prototypes automatically from unlabeled data. Based on the local-consistency and the exotic-inconsistency assumptions, it stands to reason that samples along with their closest 


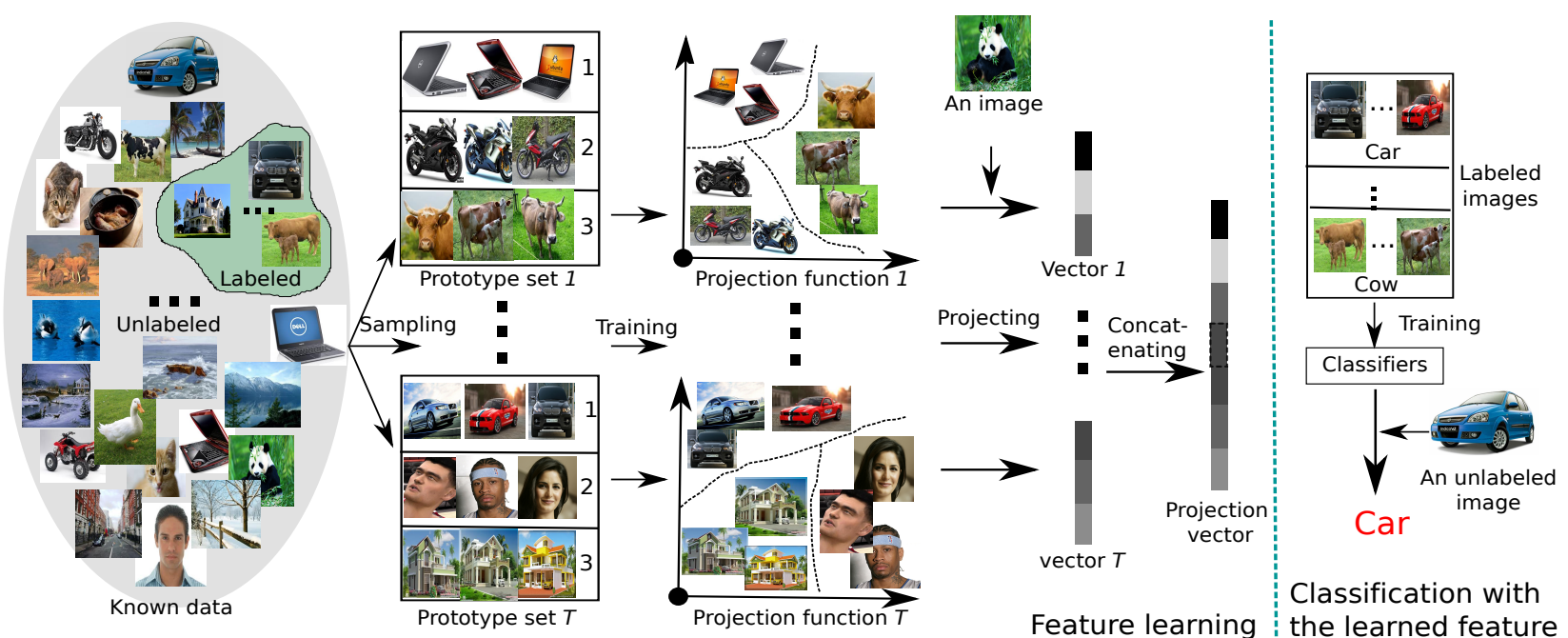

Figure 1. The pipeline of Ensemble Projection (EP). EP consists in unsupervised feature learning (left panel) and plain supervised classification (right panel). For feature learning, we sample an ensemble of $T$ diverse prototype sets from all known images and learn discriminative classifiers on them for the projection functions. Images are then projected using these functions to obtain their new representation. For classification, we train plain classifiers on labeled images with the learned features to classify the unlabeled ones.

neighbors can be "good" prototypes (defining one visual category/attribute), and far apart such prototypes can play the role of different categories. According to this observation, we design a method to sample the prototype set from all available data. Discriminative learning is then used, logistic regression in our implementation, to learn projection functions tuned to the prototypes. Images are linked to the prototypes via their projection values (classification scores). Since information carried by one single prototype set is limited and can be noisy, we borrow ideas from ensemble learning [26] to create an ensemble of diverse prototype sets, which in turn leads to an ensemble of projection functions. Images are then represented by the concatenation of their projected values (similarities) to all the image prototypes, in keeping with prototype theory [27]. We call the method Ensemble Projection (EP) and it is illustrated in Fig.1.

Solving SSL problems this way, EP addresses all the three aforementioned issues: (1) in addition to localconsistency property, it also exploits exotic-inconsistency property; (2) the learned new feature can be fed into any classifiers; and (3) it performs well for self-taught image classification, supported by experiments. Our contributions are: (1) the exotic-inconsistency assumption and solving the SSL task as a feature learning problem; (2) a simple, yet effective way to create an ensemble of diverse prototype sets; (3) experimental verification that our method is superior to competing methods, combines well with them, and is more generally applicable. While we focus in this paper on image classification, our framework is fairly general: the framework can be used for other tasks as well, such as clustering and retrieval. The code of this work is available at WWW.vision.ee.ethz.ch/ daid/EnPro.
The rest of this paper is organized as follows. S£c 2 reports on related work. S\&c 3 describes our approach, followed by experiments in S\&c 4 . S\&c 5 concludes the paper.

\section{Related Work}

Our method is generally relevant to semi-supervised learning, ensemble learning, and image feature learning.

Semi-supervised Learning. There is a large body of work on semi-supervised learning (SSL) [35]. SSL aims at enhanced learning by exploiting available, unlabeled data. One group of methods is based on label propagation over a graph, where nodes represent data examples and edges reflect their similarities. The optimal labels are those that are maximally consistent with the supervised class labels and the graph structure. Well known examples include Harmonic-Function [34], Local-Global Consistency [33], Manifold Regularization 11], and Engenfunction [10]. While having strong theoretical support, these methods cannot label unseen data. Another group of methods utilize the unlabeled data to regularize the classifying functions - enforcing the boundaries to pass through regions with a low density of data samples. The most notable methods are transductive SVM[ [13], Semi-supervised SVM [2], and semi-supervised random fores [18]. Readers are referred $t c[35]$ for a thorough overview of SSL. For semi-supervised image classification, Guillaumin et $a l[$ [11], and Shrivastava et al[. [29] presented two methods in the self-supervised manner - unlabeled images with high classification confidence are then included into the training set for the next round of learning. While obtaining promising results, they both require additional supervision $[1]$ 
needs image tags and [29] image attributes.

Ensemble Learning. Our method learns the representation from an ensemble of prototype sets, thus sharing aspects of ensemble learning (EL). EL builds a committee of base learners, and finds solutions by maximizing the agreement. Popular ensemble methods that have been extended to semi-supervised scenarios are Boosting [15] and Random Forest [18]. However, these methods still differ significantly from ours. They focus on the problem of improving classifiers by using unlabeled data. Our method learns new representations for images using all data available. Thus, it is independent of the classification methods. The reason we use EL is to capture rich visual attributes from a series of prototype sets. Other work close to ours is that of Dai et al[ $[5]$. They presented an ensemble partitioning framework for unsupervised image categorization, where weak training sets are sampled to train base learners. The whole dataset is classified by all the base learners in order to obtain a bagged proximity matrix for further clustering. A similar idea was also proposed in Random Ensemble Metric: [14], where images are projected to randomly subsampled training categories for supervised distance learning.

Feature Learning. Over the past years, a wide spectrum of features, from pixel-level to semantic-level, have been designed and used for different vision tasks. Due to the semantic gap, recent work builds up high-level features, which go beyond single images and are probably impregnated with semantic information. Notable examples are Image Attributes [8], Classemes[30], and Object Bank[19]. While getting pleasing results, these methods all require additional labeled training data, which is exactly what we want to avoid. There have been several attempt [2,3,32] to avoid the extra attribute-level supervision, but they still require canonical category-level supervision. Our representation learning is fully unsupervised. The method also shares similarity with Self-taught learning [25], where sparse coding is employed to construct higher-level features using unlabeled data. Both work attempt to leverage the regularities of general visual data to improve image representation.

\section{Our Approach}

The training data consists of both labeled data $\mathcal{D}_{l}=$ $\left\{\left(\mathbf{x}_{i}, y_{i}\right)\right\}_{i=1}^{l}$ and unlabeled data $\mathcal{D}_{u}=\left\{\mathbf{x}_{j}\right\}_{j=l+1}^{l+u}$, where $\mathbf{x}_{i}$ denotes the feature vector of image $i, y_{i} \in\{1, \ldots, K\}$ is its label, and $K$ is the number of classes. Most previous semi-supervised learning (SSL) methods learn a classifier $\phi: \mathcal{X} \mapsto \mathcal{Y}$ from $\mathcal{D}_{l}$ with a regulation term learned from $\mathcal{D}_{u}$. Our method learns a new image representation $\mathrm{f}$ from all known data $\mathcal{D}=\mathcal{D}_{l} \cup \mathcal{D}_{u}$, and train plain classifier $\phi$ on $\mathbf{f}$. $\mathbf{f}_{i}$ is a vector of similarities of image $i$ to a series of sampled image prototypes.

Assume that EP learns knowledge from $T$ prototype sets $\mathcal{P}^{t, t \in\{1, \ldots, T\}}=\left\{\left(s_{i}^{t}, c_{i}^{t}\right)\right\}_{i=1}^{r n}$, where $s_{i}^{t} \in\{1, \ldots, l+u\}$ is the index of the $i^{t h}$ chosen image, $c_{i}^{t} \in\{1, \ldots, r\}$ is the pseudo-label indicating which prototype $s_{i}^{t}$ belong to. $r$ is the number of prototypes (analogous to the number of object classes) in $\mathcal{P}^{t}$, and $n$ the number of images sampled for each prototype (e.g. $r=3$ and $n=3$ in Fiф. 1). Below, we first present our sampling method of creating a single prototype set $\mathcal{P}^{t}$ in the $t$ trial, followed by EP.

\subsection{Max-Min Sampling}

As stated, we want the prototypes to be inter-distinct and intra-compact, so that each one represents a different visual concept. To this end, we design a 2-step sampling method, termed Max-Min Sampling. The Max step is designed for the inter-distinct property, and the Min-step for the intracompact one. In particular, we first sample a skeleton of the prototype set, by looking for image candidates that are strongly spread out, i.e. at large distances from each other. We then enrich the skeleton to a prototype set by including the closest neighbors of the skeleton images. The algorithm for creating $\mathcal{P}^{t}$ is given in Algo 1 . For the skeleton, we randomly sampled $m$ hypotheses - each hypothesis consists of $r$ random sampled images - and keep the one having the largest mutual distance. This simple procedure guarantees that the sampled seed images are far from each other. Once the skeleton is created, the Min-step extends each seed image to an image prototype by introducing its $n$ nearest neighbors (including itself), in order to enrich the characteristics of each image prototype and reduce the risk of introducing noisy images. The pseudo-labels are shared by all images specifying the same prototype. It is worth pointing out that the randomized Max-step may not generate the optimal skeleton. However, it serves its purpose well. For one thing, we do not need the optimal one - we only need the prototypes to be far apart, not farthest apart. Moreover, the randomized step leaves room for randomness so that diverse visual concepts can be captured in different $\mathcal{P}^{t}$ 's. The dis(.,.) in line 5 represents the distance between two visual vectors, $L 1$ distance metric in our implementation.

\subsection{Ensemble Projection}

We now explore the use of the image prototype sets created in 331 for a new image representation. Because the prototypes are compact in feature space, each of them implicitly defines a visual concept (image attribute). This is especially true when the dataset $\mathcal{D}$ is sufficiently large, which is to be expected given the vast numbers of unlabeled images that are available. Since information carried by a single prototype set $\mathcal{P}^{t}$ is quite limited, we borrow idea from ensemble learning (EL) to create an ensemble of $T$ such sets.

As we all know, EL benefits from the precision of its base learners and their diversity. For good precision, discriminative learning method is employed as the base learner $\phi_{t}($.$) :$ 


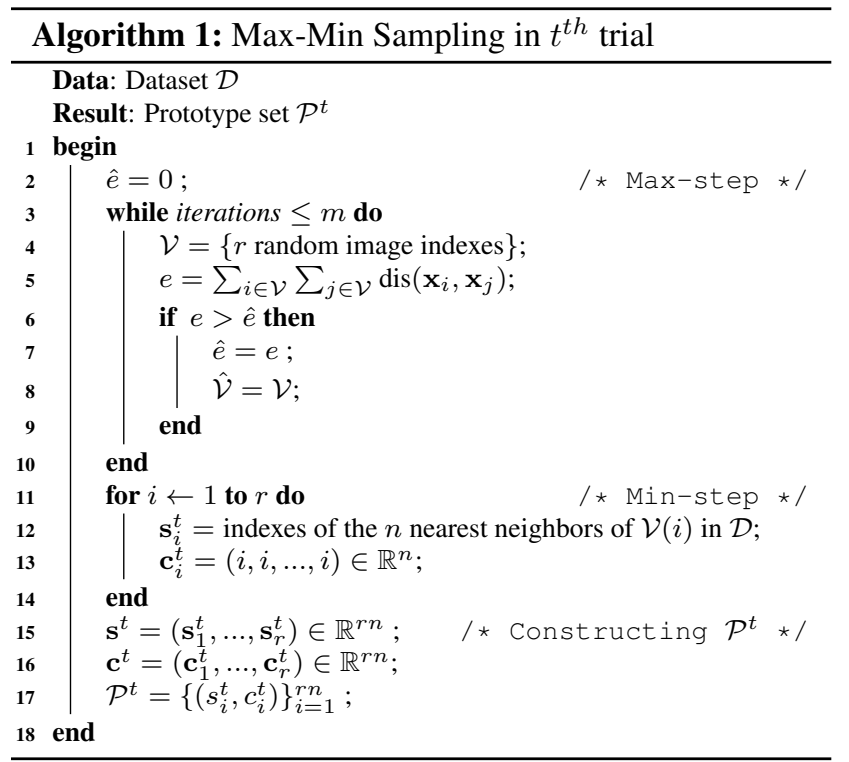

logistic regression is used in our implementation to project each input image $\mathbf{x}$ to the image prototypes to measure the similarities. For large diversity, randomness is introduced in different trials of Max-Min Sampling to create an ensemble of diverse prototype sets, so that a rich set of image attributes are captured. The vector of all similarities is then concatenated and used as a new image representation $\mathbf{f}$ for the final classification. A plain classifier (e.g. SVMs and boosting) can then be trained on $\mathcal{D}_{l}$ for our semi-supervised classification, as unlabeled data has already been explored in obtaining $\mathbf{f}$. The whole procedure of EP is presented in $\mathrm{Alg}$ - 2. Up to now, the whole pipeline in Fig 1 has been explained.

\section{Experiments}

Datasets: We evaluated our method on four datasets: Scene-15 (S-15) [17], LandUse-21 (L-21) [31], Texture-25 (T-25)[16], and Caltech-101 (C-101) 19]. Scene-15 dataset contains 15 scene categories with both indoor and outdoor environments, 4485 images in total. Each category has 200 to 400 images. LandUse- 21 consists of satellite images from 21 categories, 100 images each. Texture-25 dataset contains 25 texture categories, 40 samples each. Caltech101 contains 101 object categories, 8677 images in total, and each one has 31 to 800 images. Furthermore, we collected a random image collection by sampling 20,000 images randomly from ImageNet datase [6] to evaluate our method on the task of self-taught image classification. Since the current version of ImageNet has already had 21841 synsets (categories) and more than 14 millions of images in total, the chance is vanishingly small that images of the random image collection and images of the four datasets

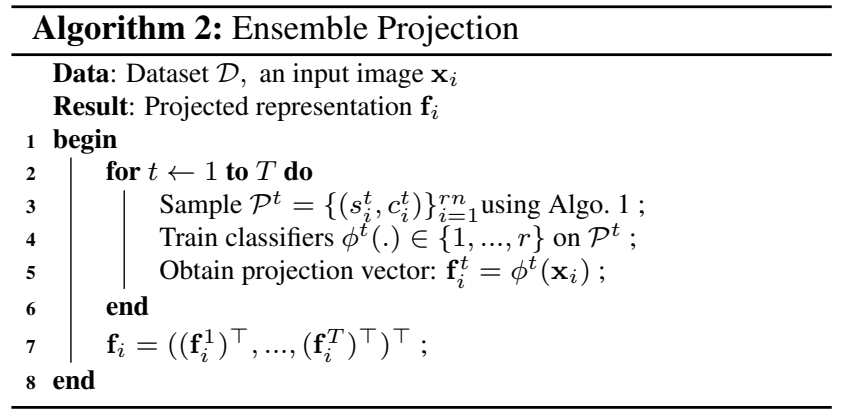

considered are coming from the same distribution.

Features: The following three features were used in our experiments: GIST [23], Pyramid of Histogram of Oriented Gradients (PHOG) $\square 3]$, and Local Binary Patterns (LBP [2, 2]. GIST was computed on the rescaled images of $256 \times 256$ pixels, in 4,8 and 8 orientations at 3 scales from coarse to fine. PHOG was computed with a 2-layer pyramid and in 8 directions. For LBP, the uniform LBP was used. These features were used due to their low dimension, as our method requires 'meaningful' neighborhoods to exploit.

Competing methods: Four classifiers were adopted to evaluate the method, with two inductive classifiers logistic regression (LR) and linear SVMs, and two transductive classifiers Harmonic-Function (HF) [34] and LapSVM (LSVM \1]. HF formulates the SSL learning problem as a Gaussian Random Field on a graph for label propagation. LapSVM extends SVMs by including a smoothness penalty term defined on the Laplacian graph. Since our method builds up a new feature representation, we illustrate the performance of all methods working with normal features and our learned features.

Experimental settings: We conducted five sets of experiments: (1) compare our method with competing methods for semi-supervised image classification, where the unlabeled images are from the same categories as the labeled ones; (2) evaluate the robustness of our method against its parameters; (3) evaluate the robustness of our method against the choices of different image features; (4) evaluate the robustness of the method against classifier models; and (5) evaluate the performance of our method for the task of self-taught image classification. For all experimental sets except (4), the same set of parameters were used for all the classifiers. We used L2-regularized LR of LIBLINEAR [7] with $C=15$ and the linear SVMs of LIBSVM [4] with $C=15$. For LapSVM, we used the scheme suggested by $\amalg 1]: \gamma_{A}$ was set as the inductive model, 10 in our case, and $\gamma_{I}$ was set as $\frac{\gamma_{I} l}{(l+u)^{2}}=100 \gamma_{A} l$.

As to features, while Alg $\phi .1$ and $\operatorname{Alg} \phi .2$ use the same notation $\mathbf{x}$, we used GIST for $A \lg \phi .1$ and the concatenation of all the three features for $A \lg \phi$. 2. This is because 

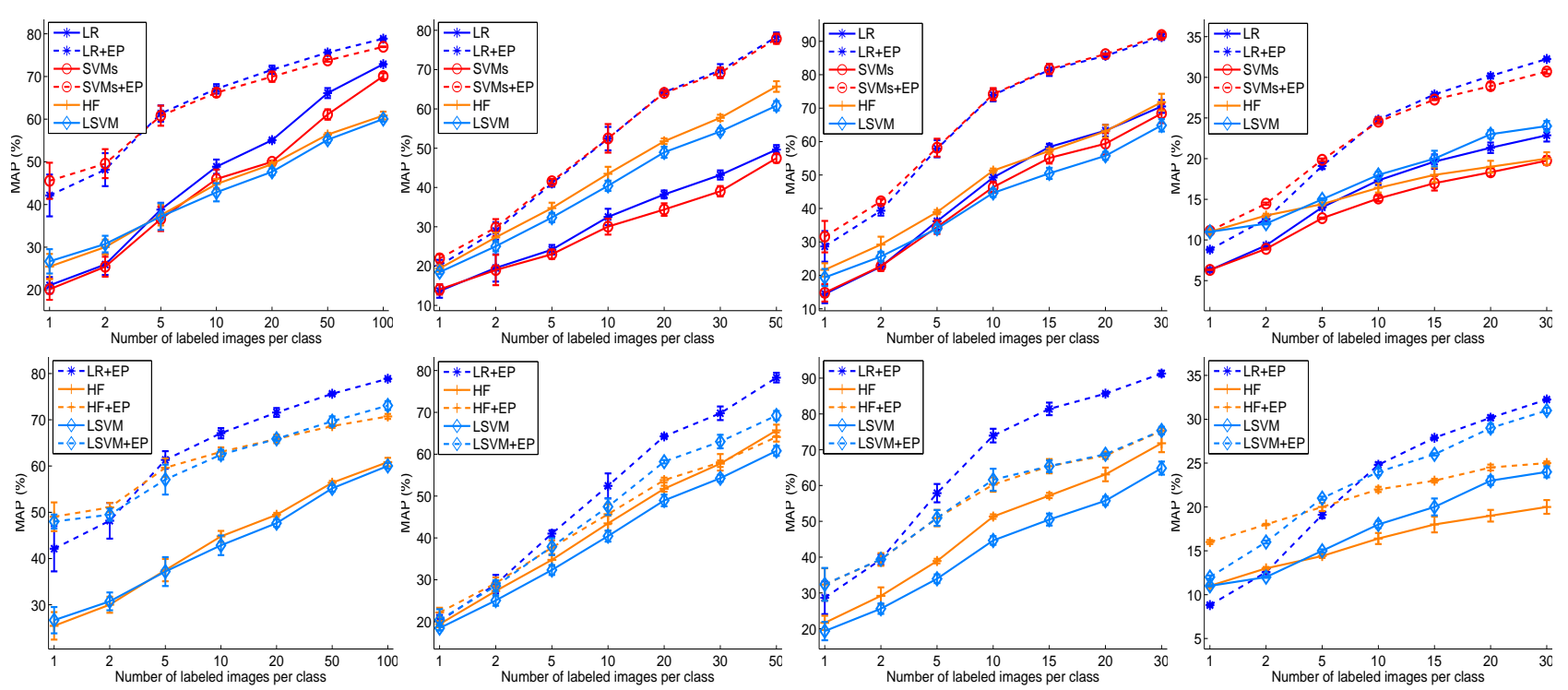

Scene-15

LandUse-21

Texture-25

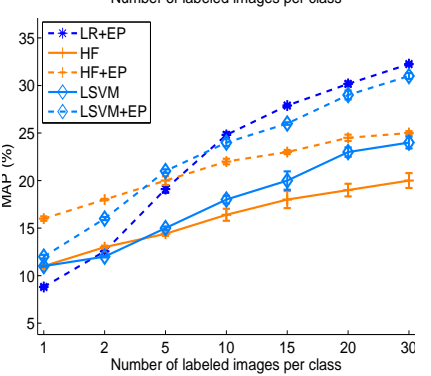

Caltech-101

Figure 2. Semi-supervised classification results on the four datasets. The top panel evaluate the performance of our learned features when fed into LR and SVMs. The bottom shows its performance when fed into HF [34] and LapSVM 1 1]. All methods were tested with two feature inputs: the concatenation of GIST, PHOG and LBP, and our learned feature from them (indicated by “+ EP”).

\begin{tabular}{|c||c|c|c|c|}
\hline Methods & S-15 & L-21 & T-25 & C-101 \\
\hline \hline LR & $38.6(1.6)$ & $24.2(1.2)$ & $36.2(2.5)$ & $14.0(0.2)$ \\
LR + EP & $\mathbf{6 1 . 3 ( 1 . 9 )}$ & $41.0(0.8)$ & $57.9(2.5)$ & $19.1(0.4)$ \\
\hline SVMs & $36.6(2.8)$ & $23.1(1.1)$ & $34.4(2.1)$ & $12.7(0.1)$ \\
SVMs + EP & $60.8(2.3)$ & $\mathbf{4 1 . 6 ( 0 . 6 )}$ & $\mathbf{5 8 . 2 ( 2 . 6 )}$ & $19.9(0.2)$ \\
\hline HE [34] & $37.5(2.4)$ & $34.8(1.4)$ & $38.9(0.6)$ & $14.4(0.3)$ \\
HF + EP & $59.6(2.0)$ & $37.7(1.7)$ & $50.9(2.2)$ & $20.0(0.4)$ \\
\hline LSVM[1] & $37.2(2.1)$ & $32.4(1.2)$ & $34.0(1.2)$ & $15.0(0.3)$ \\
LSVM + EP & $57.1(2.2)$ & $37.9(2.0)$ & $51.0(2.0)$ & $\mathbf{2 1 . 0 ( 0 . 5 )}$ \\
\hline
\end{tabular}

Table 1. MAP of semi-supervised classification on the four datasets, with 5 training examples per class. All methods were tested with two feature inputs: the concatenation of GIST, PHOG, and LBP, and our learned feature from it (indicated by "+ EP")

Alg. 1 needs a low dimensional feature to define neighborhoods, while Alg. 2 needs a discriminative feature to learn precise projection functions. Experimental set (3) was conducted by providing the same single feature to Alg. 1 and $A \lg \phi$. 2. As to the parameters of our method, we used the following for experimental sets (1), and (3)-(5): $T=300$, $r=30, n=6$, and $m=50$. A wide variety of values for them were tested in experimental set (2). For all the experiments, we perform $K$ rounds of binary classification, each time taking one class as positive and the rest as negative, as LapSVM only work for two-class cases. Multi-class average precision (MAP) was used as the evaluation criteria: the average precision over all recall values and over all classes.

\subsection{Semi-supervised Image Classification}

In this section, we evaluate all methods across all datasets for semi-supervised image classification.
Different numbers of training images per category were tested: Scene-15 with $\{1,2,5,10,20,50,100\}$, LandUse-21 with $\{1,2,5,10,20,30,50\}$, Texture-25 with $\{1,2,5,10,15,20,30\}$, and Caltech-101 with $\{1,2,5,10,15,20,30\}$. In all cases, the rest images were taken as unlabeled training data (also used for evaluation). The reported results are the average performance over 5 runs with random labeled-unlabeled splits.

Fi@. 2 shows all the results and Table 1 lists the results obtained with 5 labeled training images per class. From the top panel of Fiø. 2, it is easy to observe that the two plain classifiers LR and SVMs working with our feature perform better than the two sophisticated SSL methods LapSVM and Harmonic-Function working with the original feature, while having comparable variance. This suggests that our method can achieve promising results for semi-supervised image classification, even combined with plain classifiers. The advantages can be ascribed to two factors: (1) in addition to the local-consistency assumption, our method also exploits the exotic-inconsistency assumption; (2) the discriminative projections abstract high-level attributes from the sampled prototypes, e.g. owning "yellow-smooth" more than "dark-structured". As already proven in fully supervised scenarios $\square 8,24]$, prototype-linked, attribute-based features are very helpful for image classification. Note that our feature are learned exactly from the original feature, but going beyond one single image.

We further investigate the complementarity of our learned feature and other SSL methods for semi-supervised classification. It is interesting to see from the bottom panel 

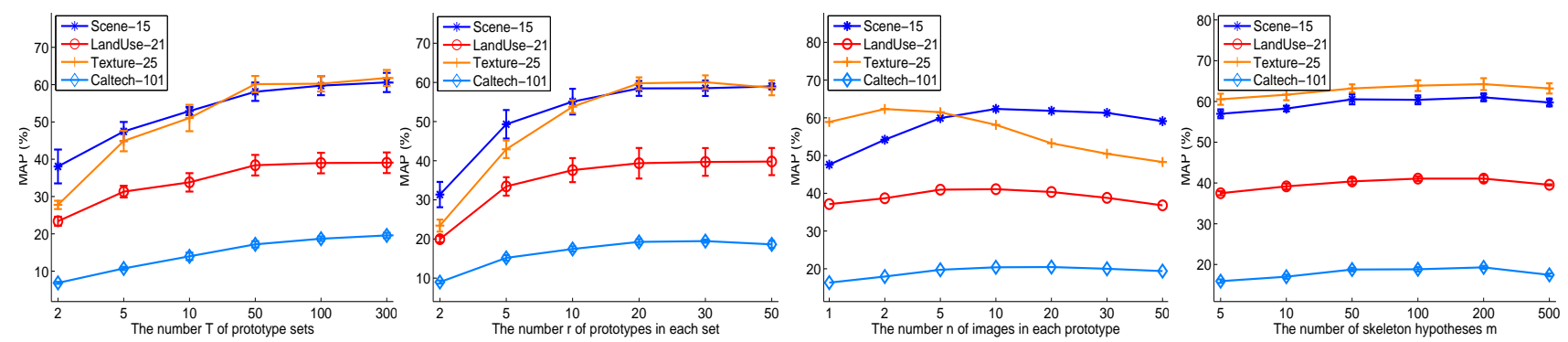

Figure 3. Performance of our method as a function of $T, r, n$, and $m$. LR was employed with 5 labeled training images per class.
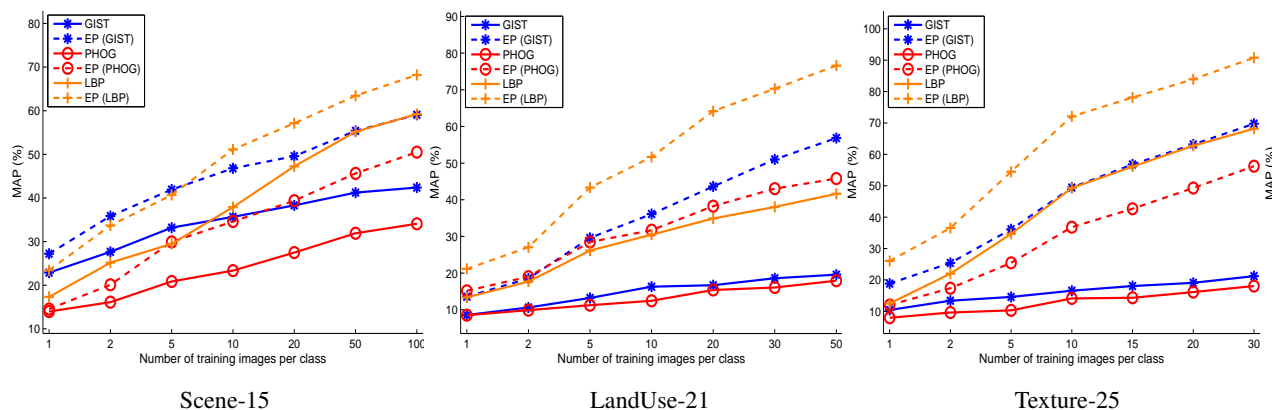

Texture-25

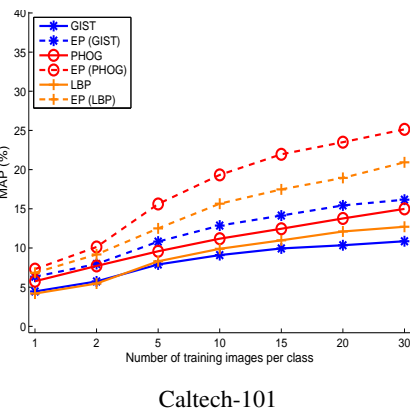

Figure 4. Comparison of our learned features (indicated by EP(.)) to the corresponding original features GIST, PHOG, and LBP . LR was used as the classifier with 5 labeled training images per class.

of Fig. 2 and Tabe 1 that combining the two boosts the performance also. This suggests that our scheme of exploiting unlabeled data and the previous ones doing so capture complementary information. The increase is more pronounced for Harmonic-Function (HF) than for LapSVM. This is in line with our intuitive understanding that HF's underlying technique label propagation on Gaussian Random Fields is more complementary to our technique discriminative learning on image neighborhoods.

\subsubsection{Robustness Against Parameters}

In this section, we examine the influence of the parameters of our method on classification performance. They are the total number of prototype sets $T$, the number of prototypes in each set $r$, the number of images in each prototype $n$, and the number of skeleton hypotheses $m$ used in Max-Min Sampling. LR was used as the classifier here. The parameters were evaluated in the following way - each time the value of one changes while the others being fixed to the values described in the experimental settings.

Fig. 3 shows the results over a range of their values. The figure shows that the performance of our method increases pretty fast with $T$, but then stabilizes quickly. It implies that the method benefits from exploiting more "novel" visual attributes (image prototypes). After $T$ increases to some threshold (e.g. 50 for the four datasets), the then exploited attributes have already been in, thus stopping boosting the performance much. For $r$, the figure shows that the performance generally increases with it. This is because a large $r$ leads to a precise attribute assignment, as a thorough comparison is performed. However, we found that when $r$ goes over 20 , the increase is not worth the computing time. A large $r$ would lead to confusing attributes, because prototypes may start overlapping with each other. For $n$, a similar trend was obtained - as $n$ increases, the characteristics of the prototypes are enriched, thus boosting the performance. But beyond some threshold (e.g. 10 in our experiments), more noisy images are introduced, thus degrading the performance. For $m$, Fis. 3 shows that an undue large one degrades the performance. This can be explained from the perspective of ensemble learning (EL). EL benefits from the strength of its base learners and their diversity. Too large an $m$ brings all prototype skeletons close the the optimal one, thus decreasing the diversity of sampled prototype sets.

Although the performance of EP will be affected by the choice of its parameters, we can see from Fiф. 3 that each of the parameters has a wide range of reasonable values to choose from. It is not difficult to choose a set of parameter values that produce better results than competing methods (c.f. Fig. 3 and Tabe1). Also, the parameters are quite intuitive and their roles are similar to the parameters of some principled methods, e.g. analogues of $m, n$ and $T$ can be found in RANSAC, $k$-NN, and Bagging, respectively.

\subsubsection{Robustness Against Features}

In this section, we elaborate the performance of our method by using different single image features, in order to see its robustness against different feature choices. The LR was 


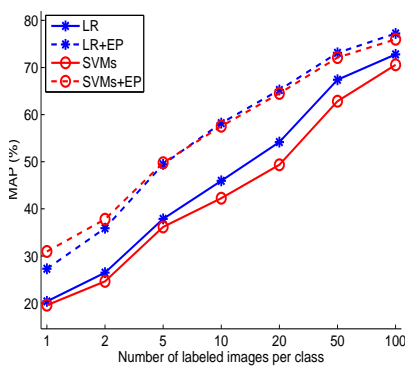

Scene- 15

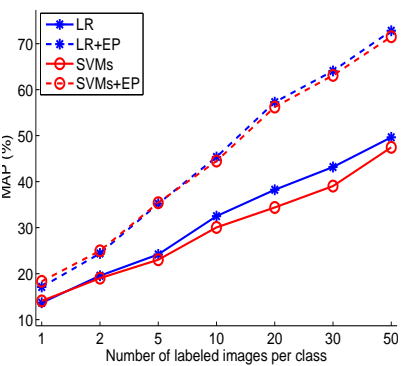

LandUse-21

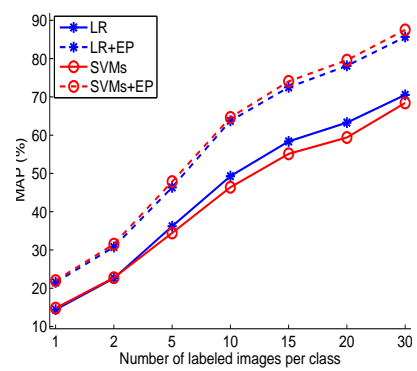

Texture-25

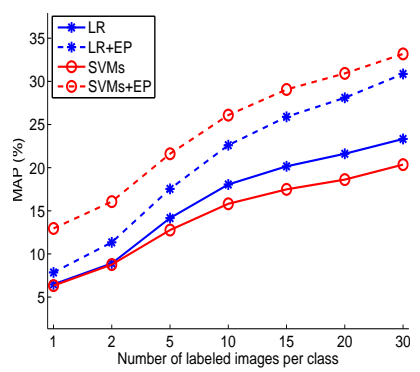

Caltech-101

Figure 5. Self-taught classification results on the four datasets. The classifiers were tested with two feature inputs: the concatenation of GIST, PHOG, and LBP, and our learned feature from it (indicated by “+ EP”).

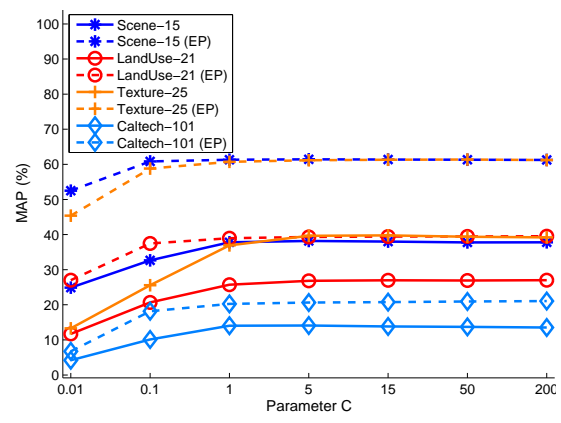

Figure 6. Comparison of our learned feature with the normal image feature against different LR models.

again used as the classifier and we compared our learned feature with the corresponding original ones, namely the GIST, the PHOG, and the LBP. The results in Fi⿻日. 4 show that all the learned features perform consistently better than the original ones, suggesting EP is robust against the choices of image features.

\subsubsection{Robustness Against Classifier Models}

In this section, we evaluate the robustness of our learned features against classifier models. Different values of the error-margin balancing parameter $C$ were tested for LR and SVMs. 5 labeled training examples per class were used. A set of values $\{0.01,0.1,1,5,15,50,200\}$ were tested for the $C$ of the SVMs and LR. The results of SVMs are not affected by the changes of $C$, probability because SVMs clearly separate the small number of training examples. Thus we only show the results of LR in Fig. 6. The figure shows that our feature consistently outperforms the original one over different classifier models. This property is important for SSL, as labeled data is limited and probably cannot accommodate a model selection technique such as Cross-Validation.

\subsection{Self-taught Image Classification}

In order to evaluate the applicability of our method, we tested it in a more general scenario, where the unlabeled data is the set of 20,000 random images from ImageNet. Projection functions were learned from images in this set plus the labeled training images in corresponding evaluation dataset, and performance was measured on the unlabeled images. Fi\$. 5 shows the classification performance with different numbers of labeled training images per class, and Table 2 lists that when 5 training images per class is used. From the figure and table, it can be found that our learned feature from the random image collection still outperforms the original feature. This property is important for semi-supervised learning, as it is often the case that one has no prior access to the data to be classified. The success could be ascribed to the fact that the "universal visual world" (the random image collection) contains abundant high-level, valuable visual attributes such as "blue and open" in some image clusters and "textured and man-made" in others. Exploiting these "hidden" visual attributes is very beneficial for narrowing down the semantic gap between low-level features and high-level classification tasks.

From the figure, we can also find that as the number of labeled training images increases, the advantage of our learned feature may decrease. It comes without much surprise as the method is designed to improve classification systems by exploiting 'unknowledgeable' (unlabeled) data. Therefore, when a sufficient number of labeled images are available, introducing additional unlabeled ones may hurt the system. This is a general, open problem for semisupervised learning (self-taught learning $\sqrt{[20]}$. One possible solution is to study when the classification systems should switch from semi-supervised learning to fully supervised learning.

\section{Conclusion}

This paper has tackled the problem of semi-supervised image classification from a novel perspective - rather than regularizing classifying functions like previous methods, we learn a new, high-level image representation. We proposed as novel concept the exotic-inconsistency assumption and designed a simple, yet effective feature learning method to use it along with local-consistency to exploit the avail- 


\begin{tabular}{|c||c|c|c|c|}
\hline Methods & S-15 & L-21 & T-25 & C-101 \\
\hline \hline LR & 37.8 & 24.2 & 36.1 & 14.2 \\
LR + EP & 49.5 & 35.2 & 46.3 & 17.5 \\
\hline SVMs & 36.2 & 23.0 & 34.4 & 12.8 \\
SVMs + EP & $\mathbf{4 9 . 8}$ & $\mathbf{3 5 . 4}$ & $\mathbf{4 7 . 0}$ & $\mathbf{1 7 . 6}$ \\
\hline
\end{tabular}

Table 2. MAP of self-taught classification, with 5 training examples per class. All methods were tested with two feature inputs: the concatenation of GIST, PHOG, and LBP and our learned feature from the 20,000 random image collection (indicated by "+ EP").

able data. By doing so, images are represented with their affinities to a rich set of discovered image attributes for classification. Extensive experiments showed that our method outperforms competing methods for semi-supervised image classification, combines well with them, and is more generally applicable.

Acknowledgements. The authors gratefully acknowledge support from the Advanced Grand VarCity and the bilateral collaboration with Toyota.

\section{References}

[1] M. Belkin, P. Niyogi, and V. Sindhwani. Manifold regularization: A geometric framework for learning from labeled and unlabeled examples. JMLR, 7(36):2399-2434, 2006.

[2] K. P. Bennett and A. Demiriz. Semi-supervised support vector machines. In NIPS, 1998.

[3] A. Bosch, A. Zisserman, and X. Muoz. Image classification using random forests and ferns. In ICCV, 2007.

[4] C.-C. Chang and C.-J. Lin. LIBSVM: A library for support vector machines. ACM Transactions on Intelligent Systems and Technology, 2:1-27, 2011.

[5] D. Dai, M. Prasad, C. Leistner, and L. V. Gool. Ensemble partitioning for unsupervised image categorization. In ECCV, 2012.

[6] J. Deng, W. Dong, R. Socher, L.-J. Li, K. Li, and L. Fei-Fei. ImageNet: A Large-Scale Hierarchical Image Database. In CVPR, 2009.

[7] R.-E. Fan, K.-W. Chang, C.-J. Hsieh, X.-R. Wang, and C.-J. Lin. Liblinear: A library for large linear classification. $J$. Mach. Learn. Res., 9:1871-1874, 2008.

[8] A. Farhadi, I. Endres, D. Hoiem, and D. Forsyth. Describing objects by their attributes. In CVPR, 2009.

[9] L. Fei-Fei, R. Fergus, and P. Perona. Learning generative visual models from few training examples: an incremental Bayesian approach tested on 101 object categories. In CVPR, WS, 2004.

[10] R. Fergus, Y. Weiss, and A. Torralba. Semi-supervised learning in gigantic image collections. In NIPS, 2009.

[11] M. Guillaumin, J. J. Verbeek, and C. Schmid. Multimodal semi-supervised learning for image classification. In $C V P R$, 2010.

[12] P. Jain and A. Kapoor. Active learning for large multi-class problems. In CVPR, 2009.

[13] T. Joachims. Transductive inference for text classification using support vector machines. In ICML, 1999.
[14] T. Kozakaya, S. Ito, and S. Kubota. Random ensemble metrics for object recognition. In ICCV, 2011.

[15] P. Kumar Mallapragada, R. Jin, A. Jain, and Y. Liu. Semiboost: Boosting for semi-supervised learning. TPAMI, 31(11):2000-2014, 2009.

[16] S. Lazebnik, C. Schmid, and J. Ponce. A sparse texture representation using local affine regions. TPAMI, 27(8):12651278, 2005.

[17] S. Lazebnik, C. Schmid, and J. Ponce. Beyond bags of features: Spatial pyramid matching for recognizing natural scene categories. In CVPR, 2006.

[18] C. Leistner, A. Saffari, J. Santner, and H. Bischof. Semisupervised random forests. In ICCV, 2009.

[19] L.-J. Li, H. Su, E. P. Xing, and F.-F. Li. Object bank: A highlevel image representation for scene classification \& semantic feature sparsification. In NIPS, 2010.

[20] Y.-F. Li and Z.-H. Zhou. Towards making unlabeled data never hurt. In ICML, 2011.

[21] X. Liu, X. Yuan, S. Yan, and H. Jin. Multi-class semisupervised svms with positiveness exclusive regularization. In $I C C V, 2011$.

[22] T. Ojala, M. Pietikäinen, and T. Mäenpää. Multiresolution gray-scale and rotation invariant texture classification with local binary patterns. TPAMI, 24(7):971-987, 2002.

[23] A. Oliva and A. Torralba. Modeling the shape of the scene: A holistic representation of the spatial envelope. IJCV, 42(3):145-175, 2001.

[24] A. Quattoni, M. Collins, and T. Darrell. Transfer learning for image classification with sparse prototype representations. In CVPR, 2008.

[25] R. Raina, A. Battle, H. Lee, B. Packer, and A. Y. Ng. Selftaught learning: transfer learning from unlabeled data. In ICML, 2007.

[26] L. Rokach. Ensemble-based classifiers. Artificial Intelligence Review, 33(1-2):1-39, 2010.

[27] E. Rosch. Principles of categorization. Cognition and Categorization, pages 27-48, 1978.

[28] V. Sharmanska, N. Quadrianto, and C. Lampert. Augmented attribute representations. In ECCV. 2012.

[29] A. Shrivastava, S. Singh, and A. Gupta. Constrained semisupervised learning using attributes and comparative attributes. In ECCV, 2012.

[30] L. Torresani, M. Szummer, and A. Fitzgibbon. Efficient object category recognition using classemes. In ECCV, 2010.

[31] Y. Yang and S. Newsam. Bag-of-visual-words and spatial extensions for land-use classification. In ACM GIS, 2010.

[32] F. X. Yu, L. Cao, R. S. Feris, J. R. Smith, and S.-F. Chang. Designing category-level attributes for discriminative visual recognition. In $C V P R, 2013$.

[33] D. Zhou, O. Bousquet, T. N. Lal, J. Weston, and B. Schlkopf. Learning with local and global consistency. In NIPS, 2004.

[34] X. Zhu, Z. Ghahramani, and J. Lafferty. Semi-supervised learning using gaussian fields and harmonic functions. In ICML, 2003.

[35] X. Zhu and A. B. Goldberg. Introduction to Semi-Supervised Learning. 2009. 